TRANSACTIONS OF THE

AMERICAN MATHEMATICAL SOCIETY

Volume 352, Number 3, Pages 1113-1129

S $0002-9947(99) 02282-5$

Article electronically published on July 7, 1999

\title{
THE SECOND BOUNDED COHOMOLOGY OF AN AMALGAMATED FREE PRODUCT OF GROUPS
}

\author{
KOJI FUJIWARA
}

Dedicated to Professor John Stallings for his 60th birthday

\begin{abstract}
We study the second bounded cohomology of an amalgamated free product of groups, and an HNN extension of a group. As an application, we show that a group with infinitely many ends has infinite dimensional second bounded cohomology.
\end{abstract}

\section{INTRODUCTION}

The bounded cohomology was defined by F. Trauber for groups and by Gromov for spaces. We review the definition of the bounded cohomology of a group $G$. Let

$$
C_{b}^{k}(G ; A)=\left\{f: G^{k} \rightarrow A \mid f \text { has bounded range }\right\},
$$

where $A=\mathbb{Z}$ or $\mathbb{R}$. The boundary $\delta: C_{b}^{k}(G ; A) \rightarrow C_{b}^{k+1}(G ; A)$ is given by

$$
\begin{aligned}
\delta f\left(g_{0}, \ldots, g_{k}\right)= & f\left(g_{1}, \ldots, g_{k}\right)+\sum_{i=1}^{k}(-1)^{i} f\left(g_{0}, \ldots, g_{i-1} g_{i}, \ldots, g_{k}\right) \\
& +(-1)^{k+1} f\left(g_{0}, \ldots, g_{k-1}\right) .
\end{aligned}
$$

The cohomology of the complex $\left\{C_{b}^{k}(G ; A), \delta\right\}$ is the bounded cohomology of $G$, denoted by $H_{b}^{*}(G ; A)$. See [G], [I] as general references for the theory of the bounded cohomology.

For any group $G$, the first bounded cohomology $H_{b}^{1}(G ; A)$ is trivial. If $G$ is amenable, then $H_{b}^{n}(G ; \mathbb{R})$ is trivial for all $n \geq 1$. The first example of a group with non-trivial second bounded cohomology was obtained by Brooks [B]. He showed that a free group of rank greater than 1 has infinite dimensional second bounded cohomology. Grigorchuk investigated the structure of the second bounded cohomology of free groups, torus knot groups and surface groups [Gr]. Yoshida [Y] and Soma [So1], [So2] studied the third bounded cohomology of surfaces and hyperbolic 3-manifolds. Epstein and the author showed that a non-trivial word-hyperbolic group has infinite dimensional second bounded cohomology [EF].

In order to state our results, we recall that $l^{1}$ denotes the Banach space of summable sequences of real numbers with the norm $\left\|\left(x_{i}\right)\right\|=\sum_{i=1}^{\infty}\left|x_{i}\right|$. It is

Received by the editors February 20, 1996 and, in revised form, November 7, 1997.

1991 Mathematics Subject Classification. Primary 20F32; Secondary 55U99, 20E06.

Key words and phrases. Bounded cohomology, ends of groups.

Most of the work was done when the author visited MSRI supported in part by NSF grant DMS-9022140 and a JSPS grant. He is supported in part by The Inamori Foundation. 
well known that the $\mathbb{R}$-vector space $l^{1}$ has dimension equal to the cardinal of the continuum.

In the case of an amalgamated free product of groups, we have the following.

Theorem 1.1. Let $G=A *_{C} B$. If $|C \backslash A / C| \geq 3$ and $|B / C| \geq 2$, then there is an injective $\mathbb{R}$-linear map $\omega: l^{1} \rightarrow H_{b}^{2}(G ; \mathbb{R})$. In particular, the dimension of $H_{b}^{2}(G ; \mathbb{R})$ as a vector space over $\mathbb{R}$ is the cardinal of the continuum.

Corollary 1.1. Let $G=A * B$ with $A \neq\{1\}, B \neq\{1\}$. If $G \neq \mathbb{Z}_{2} * \mathbb{Z}_{2}$, then there is an injective $\mathbb{R}$-linear map $\omega: l^{1} \rightarrow H_{b}^{2}(G ; \mathbb{R})$. In particular, the dimension of $H_{b}^{2}(G ; \mathbb{R})$ as a vector space over $\mathbb{R}$ is the cardinal of the continuum.

Remark. (1) Corollary 1.1 generalizes the Brooks' result on free groups.

(2) Since $\mathbb{Z}_{2} * \mathbb{Z}_{2}$ is amenable, $H_{b}^{2}\left(\mathbb{Z}_{2} * \mathbb{Z}_{2} ; \mathbb{R}\right)$ is trivial.

Corollary 1.2. Let $G=A *_{C} B$. If $|A|=\infty,|C|<\infty$, and $|B / C| \geq 2$, then there is an injective $\mathbb{R}$-linear map $\omega: l^{1} \rightarrow H_{b}^{2}(G ; \mathbb{R})$. In particular, the dimension of $H_{b}^{2}(G ; \mathbb{R})$ as a vector space over $\mathbb{R}$ is the cardinal of the continuum.

Corollary 1.3. Let $G=A *_{C} B$. If $A$ is abelian, $|A / C| \geq 3$, and $|B / C| \geq 2$, then there is an injective $\mathbb{R}$-linear map $\omega: l^{1} \rightarrow H_{b}^{2}(G ; \mathbb{R})$. In particular, the dimension of $H_{b}^{2}(G ; \mathbb{R})$ as a vector space over $\mathbb{R}$ is the cardinal of the continuum.

Example. $P S L_{2}(\mathbb{Z})=\mathbb{Z}_{2} * \mathbb{Z}_{3}$ and $S L_{2}(\mathbb{Z})=\mathbb{Z}_{4} *_{\mathbb{Z}_{2}} \mathbb{Z}_{6}$ satisfy the assumption of Theorem 1.1. They are non-elementary word-hyperbolic groups too.

In the case of HNN extensions of groups, we obtain the following result.

Theorem 1.2. Let $G=A *_{C, \varphi}$. If $|A / C| \geq 2,|A / \varphi(C)| \geq 2$, then there is an injective $\mathbb{R}$-linear map $\omega: l^{1} \rightarrow H_{b}^{2}(G ; \mathbb{R})$. In particular, the dimension of $H_{b}^{2}(G ; \mathbb{R})$ as a vector space over $\mathbb{R}$ is the cardinal of the continuum.

Due to the Stallings' structure theorem in $[\mathrm{S}]$ on a group with infinitely many ends, Theorems 1.1 and 1.2 imply the following.

Theorem 1.3. If $G$ is a finitely generated group with infinitely many ends, then there is an injective $\mathbb{R}$-linear map $\omega: l^{1} \rightarrow H_{b}^{2}(G ; \mathbb{R})$. In particular, the dimension of $H_{b}^{2}(G ; \mathbb{R})$ as a vector space over $\mathbb{R}$ is the cardinal of the continuum.

Theorems 1.1 and 1.2 sometimes enable us to decide the second bounded cohomologies of spaces which decompose along (codimension one) subspaces. For example if a 3-manifold $M$ is Haken, with an incompressible embedded surface $S, \pi_{1}(M)$ decomposes along $\pi_{1}(S)$. In the case $S$ is separating/non-separating, then Theorem 1.1/1.2 (respectively) may apply. The conditions on the numbers of (double) cosets in the theorems are not very restrictive, so these would be satisfied in this case, but the author does not know if it is always the case. In fact it is true that the second bounded cohomology of a compact, geometric (in the sense of Thurston) 3-manifold $M$ is either trivial or infinite dimensional, which depends on the geometry of $M$ [FO]. In the proof one applies Theorems 1.1 and 1.2 to the canonical decomposition of $M$ (due to Jaco-Shalen and Johannson) along embedded 2-spheres and tori. One extra ingredient in the argument is that the second bounded cohomology of the fundamental group of a complete hyperbolic manifold with finite volume is infinite dimensional $[\mathrm{F}]$.

The author would like to thank J. Stallings for his useful suggestions. He also appreciates very insightful comments by the referee. 


\section{QUASI HOMOMORPHISM AND THE COUNTING FUNCTION}

Let $G$ be a discrete group with a (finite or infinite) set of generators and $\Gamma(G)$ the Cayley graph. $\Gamma(G)$ is a path metric space with each edge of length 1 . If the generating set is infinite, $\Gamma(G)$ is not locally compact. We only consider paths which start/end at vertices of $\Gamma(G)$, whose lengths are non-negative integers. Note that the distance between two vertices of $\Gamma(G)$ is well-defined and there always exist paths which achieve the distance (i.e. geodesics).

For a word $w=x_{1} x_{2} \ldots x_{n}$ in these generators, define $|w|=n$. Let $\bar{w}$ be the element of $G$ which is represented by the word $w$. Define $w^{-1}=x_{n}^{-1} \ldots x_{1}^{-1}$. We sometimes identify a word $w$ and the path starting at 1 and labeled by $w$ in $\Gamma(G)$. For a path $\alpha$ labeled by $w$, define $|\alpha|=|w|$ and $\bar{\alpha}=\bar{w}$. For an element $g$ in $G$, define $|g|=\inf _{\alpha}|\alpha|$, where $\alpha$ ranges over all the paths with $\bar{\alpha}=g$.

Let $\alpha$ be a finite path in $\Gamma(G)$. Define $|\alpha|_{w}$ to be the maximal number of times that $w$ can be seen as a subword of $\alpha$ without overlapping. We define

$$
c_{w}(\alpha)=\sup _{\alpha^{\prime}}\left\{\left|\alpha^{\prime}\right|_{w}-\left(\left|\alpha^{\prime}\right|-|\bar{\alpha}|\right)\right\}=|\bar{\alpha}|-\inf _{\alpha^{\prime}}\left(\left|\alpha^{\prime}\right|-\left|\alpha^{\prime}\right|_{w}\right),
$$

where $\alpha^{\prime}$ ranges over all the paths with the same starting point as $\alpha$ and the same finishing point. Note that the infimum in the above definition is always attained by some paths. If $\alpha^{\prime}$ attains the infimum, we say that $\alpha^{\prime}$ realizes $c_{w}$ at $\alpha$.

Lemma 2.1. If $\alpha$ is a geodesic, then

$$
\frac{|\alpha|}{|w|} \geq c_{w}(\alpha) \geq|\alpha|_{w}
$$

Proof. Let $\alpha^{\prime}$ realize $c_{w}$ at $\alpha$. Then, since $\left|\alpha^{\prime}\right|-\left|\alpha^{\prime}\right|_{w} \leq|\alpha|-|\alpha|_{w}$, we find

$$
c_{w}(\alpha)=|\alpha|-\left(\left|\alpha^{\prime}\right|-\left|\alpha^{\prime}\right|_{w}\right) \geq|\alpha|_{w} .
$$

To show the other inequality, note that $\left|\alpha^{\prime}\right|_{w} \leq \frac{\left|\alpha^{\prime}\right|}{|w|}$. This implies

$$
\left|\alpha^{\prime}\right|-\left|\alpha^{\prime}\right|_{w} \geq\left|\alpha^{\prime}\right|-\frac{\left|\alpha^{\prime}\right|}{|w|}=\left(1-\frac{1}{|w|}\right)\left|\alpha^{\prime}\right| \geq\left(1-\frac{1}{|w|}\right)|\alpha| .
$$

Thus,

$$
c_{w}(\alpha)=|\alpha|-\left(\left|\alpha^{\prime}\right|-\left|\alpha^{\prime}\right|_{w}\right) \leq \frac{|\alpha|}{|w|}
$$

For each $g$ in $G$, we choose $\gamma_{g}$ to be a path from 1 to $g$ and set $c_{w}(g)=c_{w}\left(\gamma_{g}\right)$. Then $c_{w}(g)$ does not depend on the choice of $\gamma_{g}$. The following result is clear from the previous lemma.

Lemma 2.2. For all $g \in G$,

$$
\frac{|g|}{|w|} \geq c_{w}(g)
$$

Let $f \in C^{1}(G ; \mathbb{R})$. If there exists a constant $D<\infty$ such that

$$
|f(g h)-f(g)-f(h)| \leq D
$$

for all $g, h \in G$, then we say $f$ is a quasi-homomorphism with defect $D$. Let $f$ be a quasi-homomorphism with defect $D$. Then $|\delta f| \leq D$ and $\delta(\delta f)=0$; thus $\delta f \in Z_{b}^{2}(G ; \mathbb{R})$, which defines $[\delta f] \in H_{b}^{2}(G ; \mathbb{R})$. Note that we always have $[\delta f]=0$ 
in $H^{2}(G ; \mathbb{R})$; however, we may have $[\delta f] \neq 0$ in $H_{b}^{2}(G ; \mathbb{R})$ since $f$ is not necessarily in $C_{b}^{1}(G ; \mathbb{R})$. Now we define

$$
h_{w}=c_{w}-c_{w^{-1}} \in C^{1}(G ; \mathbb{Z}) .
$$

These 1-cochains are candidates for bounded quasi-homomorphisms (see Propositions 3.1 and 6.1).

\section{QUASI HOMOMORPHISMS ON $A *_{C} B$}

Let $G=A *_{C} B$ with $|A / C| \geq 2,|B / C| \geq 2$. Take the set $\{A \cup B\} \backslash\{1\}$ as a set of generators of $G$ and denote its Cayley graph by $\Gamma(G)$.

If a word $w=x_{1} \ldots x_{n}$ satisfies $n=1$ or $x_{1}, x_{3}, \cdots \in A \backslash C$ (or $B \backslash C$ ) and $x_{2}, x_{4}, \cdots \in B \backslash C$ (or $A \backslash C$, respectively), then we say $w$ is reduced.

Lemma 3.1. A word $w=x_{1} \ldots x_{n}$ is reduced if and only if it is a geodesic in $\Gamma(G)$.

Proof. Assume $w$ is not reduced; then there exists a subword $x_{i} x_{i+1}$ with $x_{i}, x_{i+1} \in$ $A$ (or $x_{i}, x_{i+1} \in B$ ). Then $\overline{x_{i} x_{i+1}} \in A$ (or $B$, respectively); thus $\left|\overline{x_{i} x_{i+1}}\right| \leq 1$. Therefore $x_{i} x_{i+1}$ is not a geodesic; hence $w$ is not a geodesic.

On the other hand, assume $w$ is not a geodesic. To show that $w$ is not reduced by contradiction, suppose $w$ is reduced. Take a geodesic $\gamma$ such that $\bar{w}=\bar{\gamma}$. Note that $\gamma$ is reduced. Then $|w|=|\gamma|$ since reduced words representing a common element have the same length. Thus $w$ is a geodesic. This is a contradiction.

Lemma 3.2. Let $w$ be a word such that $w^{2}$ is reduced. Let $\alpha$ be a path. Then there is a geodesic which realizes $c_{w}$ at $\alpha$.

Proof. Since $w^{2}$ is reduced, $w$ is reduced. Let $\gamma$ be a path which realizes $c_{w}$ at $\alpha$ such that $|\gamma|_{w}$ is minimal among all the realizing paths at $\alpha$. We claim that $\gamma$ is a geodesic. Indeed, if $|\gamma|_{w}=0$, then $\gamma$ is a geodesic. Suppose $|\gamma|_{w}=n>0$. Then $\gamma$ is written such that

$$
\gamma_{1} w_{1} \gamma_{2} \ldots w_{n} \gamma_{n+1}
$$

where $w_{i}$ is a copy of $w$ and $\gamma_{i}$ may be an empty word. First, to show that every $\gamma_{i}$ is reduced by contradiction, suppose $\gamma_{I}$ is not reduced. Replace $\gamma_{I}$ by a reduced word $\gamma_{I}^{\prime}$ with $\overline{\gamma_{I}}=\overline{\gamma_{I}^{\prime}}$; then we have a new path

$$
\gamma^{\prime}=\gamma_{1} w_{1} \gamma_{2} \ldots \gamma_{I}^{\prime} \ldots w_{n} \gamma_{n+1},
$$

which satisfies $\left|\gamma^{\prime}\right|<|\gamma|,\left|\gamma^{\prime}\right|_{w} \geq|\gamma|_{w}$ and $\bar{\gamma}=\overline{\gamma^{\prime}}$. This is impossible since $\gamma$ is a realizing path. Thus every $\gamma_{i}$ is reduced. Next, in order to show that $\gamma$ is reduced by contradiction, suppose not. Since $w^{2}$ is reduced, there is a subword $w_{i} \gamma_{i+1} w_{i+1}$ of $\gamma$ which is not reduced and $\gamma_{i+1}$ is not empty. Since $w_{i}, w_{i+1}, \gamma_{i+1}$ are reduced, one of the following four cases occurs.

(i) The last letter of $w_{i}$ and the initial letter of $\gamma_{i+1}$ are in $A$.

(ii) The last letter of $w_{i}$ and the initial letter of $\gamma_{i+1}$ are in $B$.

(iii) The last letter of $\gamma_{i+1}$ and the initial letter of $w_{i+1}$ are in $A$.

(iv) The last letter of $\gamma_{i+1}$ and the initial letter of $w_{i+1}$ are in $B$.

Suppose (i) holds. Then

$$
w_{i}=\ldots b_{1} a_{1}, \gamma_{i+1}=a_{2} b_{2} \ldots, a_{i} \in A, b_{i} \in B .
$$

We rewrite the subword $w_{i} \gamma_{i+1}$ in $\gamma$ as

$$
w_{i} \gamma_{i+1}=\ldots b_{1} a_{1} a_{2} b_{2} \ldots=\ldots b_{1} a^{\prime} b_{2} \ldots,
$$




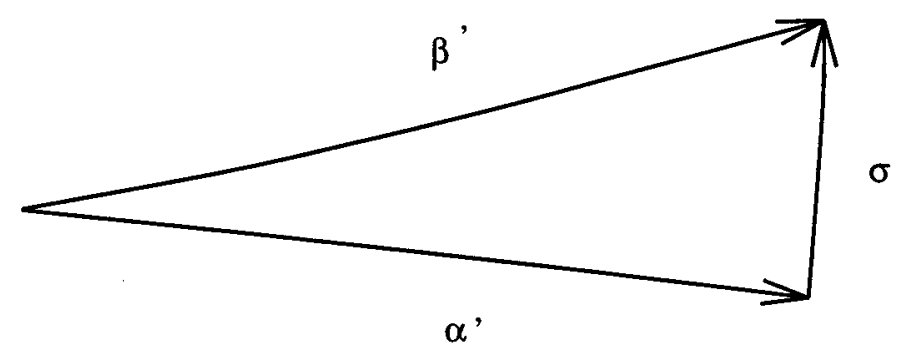

Figure 1. Geodesic triangle. This illustrates Lemma 3.3.

where $a^{\prime}=a_{1} a_{2} \in A$. This gives a new word $\gamma^{\prime}$ with $\left|\gamma^{\prime}\right|=|\gamma|-1$, and $\left|\gamma^{\prime}\right|_{w} \geq$ $|\gamma|_{w}-1$. Since $\overline{\gamma^{\prime}}=\bar{\gamma}$ and $|\gamma|-|\gamma|_{w} \geq\left|\gamma^{\prime}\right|-\left|\gamma^{\prime}\right|_{w}$, we find $\gamma^{\prime}$ is another realizing path with $\left|\gamma^{\prime}\right|_{w}<|\gamma|_{w}$. This contradicts the choice of $\gamma$. We showed that $\gamma$ is reduced. By Lemma 3.1, $\gamma$ is a geodesic. Similar argument applies to the other three cases.

Lemma 3.3. Suppose $w^{2}$ is reduced. Let $\alpha, \beta$ be paths starting at 1 . Then we have

$$
\left|c_{w}(\alpha)-c_{w}(\beta)\right| \leq 2\left|\overline{\alpha^{-1} \beta}\right|
$$

Proof. Take realizing geodesics $\alpha^{\prime}$ and $\beta^{\prime}$ of $c_{w}$ at $\alpha$ and $\beta$ respectively (see Figure 1). Then $c_{w}(\alpha)=\left|\alpha^{\prime}\right|_{w}, c_{w}(\beta)=\left|\beta^{\prime}\right|_{w}$. Take a geodesic $\sigma$ with $\overline{\alpha^{-1} \beta}=\bar{\sigma}$. Since the path $\alpha^{\prime} \sigma$ satisfies $\overline{\alpha^{\prime} \sigma}=\overline{\beta^{\prime}}$,

$$
\left|\beta^{\prime}\right|-\left|\beta^{\prime}\right|_{w} \leq\left|\alpha^{\prime} \sigma\right|-\left|\alpha^{\prime} \sigma\right|_{w} .
$$

Since $\left|\alpha^{\prime} \sigma\right|=\left|\alpha^{\prime}\right|+|\sigma|$ and $\left|\alpha^{\prime} \sigma\right|_{w} \geq\left|\alpha^{\prime}\right|_{w}+|\sigma|_{w}$,

$$
\left|\beta^{\prime}\right|-\left|\beta^{\prime}\right|_{w} \leq\left|\alpha^{\prime}\right|+|\sigma|-\left|\alpha^{\prime}\right|_{w}-|\sigma|_{w} .
$$

Thus

$$
c_{w}(\beta)=\left|\beta^{\prime}\right|_{w} \geq\left|\alpha^{\prime}\right|_{w}+\left|\beta^{\prime}\right|-\left|\alpha^{\prime}\right|-|\sigma|+|\sigma|_{w} \geq c_{w}(\alpha)-2|\sigma|,
$$

since $\left|\alpha^{\prime}\right|_{w}=c_{w}(\alpha),\left|\beta^{\prime}\right|-\left|\alpha^{\prime}\right| \geq-|\sigma|$, and $|\sigma|_{w} \geq 0$. Similarly, $c_{w}(\alpha) \geq c_{w}(\beta)-2|\sigma|$. We get $\left|c_{w}(\alpha)-c_{w}(\beta)\right| \leq 2|\sigma|=2\left|\overline{\alpha^{-1} \beta}\right|$.

Lemma 3.4. Suppose $w^{2}$ is reduced. Let $\alpha, \beta$ be paths starting at 1 . Then we have

$$
\left|h_{w}(\alpha)-h_{w}(\beta)\right| \leq 4\left|\overline{\alpha^{-1} \beta}\right| .
$$

Proof. By definition $h_{w}=c_{w}-c_{w^{-1}}$. Apply Lemma 3.3.

\section{Lemma 3.5.}

$$
c_{w}(\alpha)=c_{w^{-1}}\left(\alpha^{-1}\right)
$$

Proof. Clear from the definition of $c_{w}$.

\section{Lemma 3.6.}

$$
h_{w}(\alpha)=-h_{w}\left(\alpha^{-1}\right)
$$

Proof. By definition, $h_{w}\left(\alpha^{-1}\right)=c_{w}\left(\alpha^{-1}\right)-c_{w^{-1}}\left(\alpha^{-1}\right)=c_{w^{-1}}(\alpha)-c_{w}(\alpha)=$ $-h_{w}(\alpha)$.

Lemma 3.7. Suppose $w^{2}$ is reduced. Let $\alpha$ be a geodesic. If $\alpha=\alpha_{1} \alpha_{2}$, then

$$
\left|h_{w}(\alpha)-h_{w}\left(\alpha_{1}\right)-h_{w}\left(\alpha_{2}\right)\right| \leq 10 .
$$




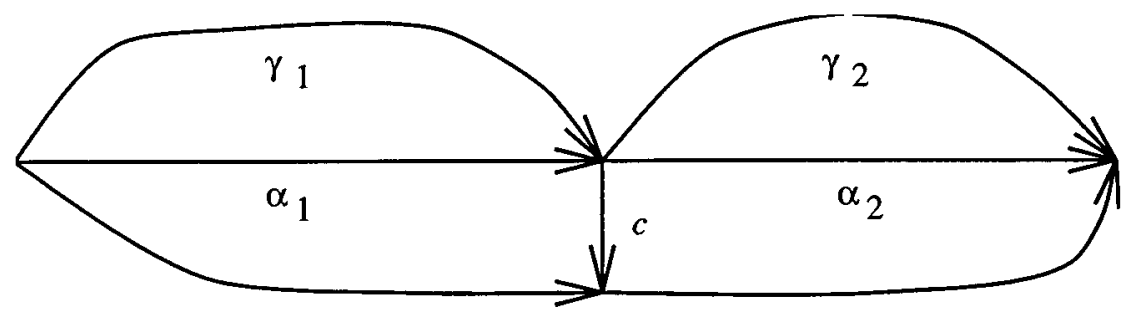

Figure 2. Dividing geodesics. This illustrates Lemma 3.7. Note $\alpha=\alpha_{1} \alpha_{2}, \gamma=\gamma_{1} \gamma_{2}$ and $\sigma=\sigma_{1} \sigma_{2}$.

Proof. We first show $\left|c_{w}(\alpha)-c_{w}\left(\alpha_{1}\right)-c_{w}\left(\alpha_{2}\right)\right| \leq 5$. Let $\gamma_{1}$ and $\gamma_{2}$ be realizing geodesics of $c_{w}$ at $\alpha_{1}$ and $\alpha_{2}$, respectively. We have $\left|\gamma_{1}\right|_{w}=c_{w}\left(\alpha_{1}\right),\left|\gamma_{2}\right|_{w}=c_{w}\left(\alpha_{2}\right)$. Set $\gamma=\gamma_{1} \gamma_{2}$; then $\bar{\gamma}=\bar{\alpha}$. As $|\alpha|=\left|\alpha_{1}\right|+\left|\alpha_{2}\right|=\left|\gamma_{1}\right|+\left|\gamma_{2}\right|=|\gamma|$, $\gamma$ is a geodesic. Since $\gamma$ is a geodesic with $\bar{\gamma}=\bar{\alpha}$, we get $c_{w}(\alpha) \geq|\gamma|_{w}$. Thus

$$
c_{w}(\alpha) \geq|\gamma|_{w} \geq\left|\gamma_{1}\right|_{w}+\left|\gamma_{2}\right|_{w}=c_{w}\left(\alpha_{1}\right)+c_{w}\left(\alpha_{2}\right) .
$$

On the other hand, take a realizing geodesic $\sigma$ at $\alpha$; then $c_{w}(\alpha)=|\sigma|_{w}$. Since $\alpha$ and $\sigma$ are reduced, there exists subdivision of $\sigma, \sigma=\sigma_{1} \sigma_{2}$ such that $\alpha_{1}^{-1} \sigma_{1}=$ $\overline{\alpha_{2} \sigma_{2}^{-1}}=c$, for some $c \in C$ (see Figure 2). Since $|c| \leq 1$, by Lemma 3.3 and 3.5, we get, for $i=1,2$,

$$
\left|c_{w}\left(\alpha_{i}\right)-c_{w}\left(\sigma_{i}\right)\right| \leq 2
$$

Since $\sigma_{1}, \sigma_{2}$ are geodesics, we see $\left|\sigma_{i}\right|_{w} \leq c_{w}\left(\sigma_{i}\right)$ for $i=1,2$. Thus

$$
c_{w}(\alpha)=|\sigma|_{w} \leq\left|\sigma_{1}\right|_{w}+\left|\sigma_{2}\right|_{w}+1 \leq c_{w}\left(\sigma_{1}\right)+c_{w}\left(\sigma_{2}\right)+1 \leq c_{w}\left(\alpha_{1}\right)+c_{w}\left(\alpha_{2}\right)+5 .
$$

We get $\left|c_{w}(\alpha)-c_{w}\left(\alpha_{1}\right)-c_{w}\left(\alpha_{2}\right)\right| \leq 5$. Similarly, we have $\mid c_{w^{-1}}(\alpha)-c_{w^{-1}}\left(\alpha_{1}\right)-$ $c_{w^{-1}}\left(\alpha_{2}\right) \mid \leq 5$. Since $h_{w}=c_{w}-c_{w^{-1}}$, we obtain $\left|h_{w}(\alpha)-h_{w}\left(\alpha_{1}\right)-h_{w}\left(\alpha_{2}\right)\right| \leq 10$.

Proposition 3.1. Let $w$ be a word. Suppose $w^{2}$ is reduced. Then $h_{w}: G \rightarrow \mathbb{Z}$ is a quasi-homomorphism whose defect is uniformly bounded by $78 ;\left|\delta h_{w}\right| \leq 78$.

Proof. Let $x, y$ be elements in $G$. By definition $\delta h_{w}(x, y)=h_{w}(x)+h_{w}(y)-h_{w}(x y)$. Let $\alpha, \beta, \gamma$ be geodesics such that $\bar{\alpha}=x, \bar{\beta}=y, \bar{\gamma}=x y$. They are reduced paths. Since $\overline{\alpha \beta}=\bar{\gamma}$ and $\alpha, \beta, \gamma$ are reduced, there exist subdivisions of $\alpha, \beta, \gamma$ such that

$$
\alpha=\alpha_{1} \alpha_{2} \alpha_{3}, \beta=\beta_{1} \beta_{2} \beta_{3}, \gamma=\gamma_{1} \gamma_{2} \gamma_{3},
$$

and that

$$
\overline{\gamma_{1}^{-1} \alpha_{1}}=c_{1}, \overline{\alpha_{3} \beta_{1}}=c_{2}, \overline{\beta_{3} \gamma_{3}^{-1}}=c_{3}
$$

for some $c_{1}, c_{2}, c_{3} \in C$ and $\overline{\alpha_{2}}, \overline{\beta_{2}}, \overline{\gamma_{2}}$ are (simultaneously) in $A$ or $B$ (see Figure 3).

Since $\overline{\alpha_{2}}$ is in $A$ or $B$, we find $c_{w}\left(\alpha_{2}\right), c_{w^{-1}}\left(\alpha_{2}\right) \leq\left|\overline{\alpha_{2}}\right| \leq 1$. Thus $\left|h_{w}\left(\alpha_{2}\right)\right| \leq 2$. Similarly, $\left|h_{w}\left(\beta_{2}\right)\right|,\left|h_{w}\left(\gamma_{2}\right)\right| \leq 2$.

Using Lemma 3.7 twice for $\alpha=\left(\left(\alpha_{1} \alpha_{2}\right) \alpha_{3}\right)$, we get

$$
\left|h_{w}(\alpha)-h_{w}\left(\alpha_{1}\right)-h_{w}\left(\alpha_{2}\right)-h_{w}\left(\alpha_{3}\right)\right| \leq 20 ;
$$

thus $\left|h_{w}(\alpha)-h_{w}\left(\alpha_{1}\right)-h_{w}\left(\alpha_{3}\right)\right| \leq 22$. Similarly,

$$
\begin{gathered}
\left|h_{w}(\beta)-h_{w}\left(\beta_{1}\right)-h_{w}\left(\beta_{3}\right)\right| \leq 22, \\
\left|h_{w}(\gamma)-h_{w}\left(\gamma_{1}\right)-h_{w}\left(\gamma_{3}\right)\right| \leq 22 .
\end{gathered}
$$




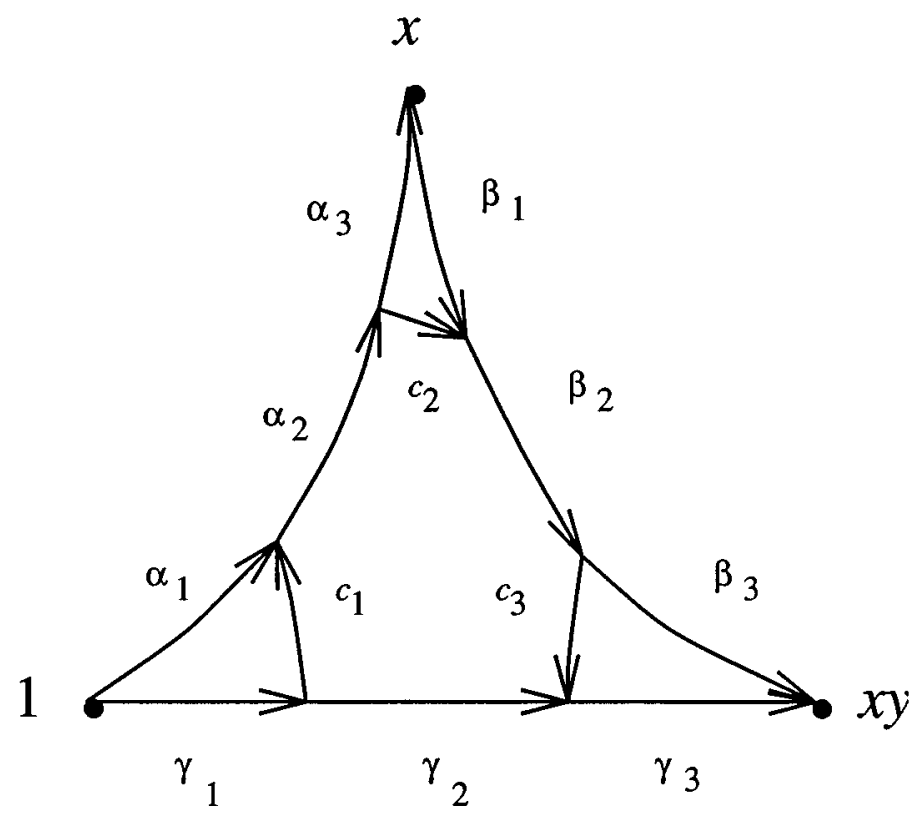

Figure 3. Dividing a geodesic triangle. This illustrates Proposition 3.1. Elements $c_{1}, c_{2}, c_{3}$ are in $C$.

Therefore, by Lemmas 3.4 and 3.6,

$$
\begin{aligned}
& \left|h_{w}(\alpha)+h_{w}(\beta)-h_{w}(\gamma)\right| \\
\leq & \left|h_{w}\left(\alpha_{1}\right)+h_{w}\left(\alpha_{3}\right)+h_{w}\left(\beta_{1}\right)+h_{w}\left(\beta_{3}\right)-h_{w}\left(\gamma_{1}\right)-h_{w}\left(\gamma_{3}\right)\right|+66 \\
\leq & \left|h_{w}\left(\alpha_{1}\right)-h_{w}\left(\gamma_{1}\right)\right|+\left|h_{w}\left(\beta_{1}\right)-h_{w}\left(\alpha_{3}^{-1}\right)\right|+\left|h_{w}\left(\gamma_{3}^{-1}\right)-h_{w}\left(\beta_{3}^{-1}\right)\right|+66 \\
\leq & 4\left(\left|c_{1}\right|+\left|c_{2}\right|+\left|c_{3}\right|\right)+66 \leq 78,
\end{aligned}
$$

since $\left|c_{i}\right| \leq 1$.

By definition, $h_{w}(\alpha)=h_{w}(x), h_{w}(\beta)=h_{w}(y)$ and $h_{w}(\gamma)=h_{w}(x y)$. This implies

$$
\left|h_{w}(x)+h_{w}(y)-h_{w}(x y)\right| \leq 78 .
$$

\section{Choice of words for $A *_{C} B$}

The goal of this section is to prove the following proposition.

Proposition 4.1. Let $G=A *_{C} B$ with $|C \backslash A / C| \geq 3$ and $|B / C| \geq 2$. Then there exist words $w_{i}, 0 \leq i<\infty$, which satisfy the following properties.

(1) For all $i \geq 0$ and all $n \geq 1, h_{w_{i}}\left(w_{i}^{n}\right)=n$.

(2) For all $j>i \geq 0$ and all $n \geq 1, h_{w_{j}}\left(w_{i}^{n}\right)=0$.

(3) For all $i \geq 0, \overline{w_{i}} \in[G, G]$.

(4) For all $i \geq 0, w_{i}^{2}$ is reduced.

(5) $\lim _{i \rightarrow \infty}\left|w_{i}\right|=\infty$.

Let $G=A *_{C} B$ with $|C \backslash A / C| \geq 3$ and $|B / C| \geq 2$. Let $w$ and $w^{\prime}$ be reduced paths which share the starting point and the finishing point as well. Let

$$
w=a_{1} b_{1} \ldots a_{n} b_{n},
$$




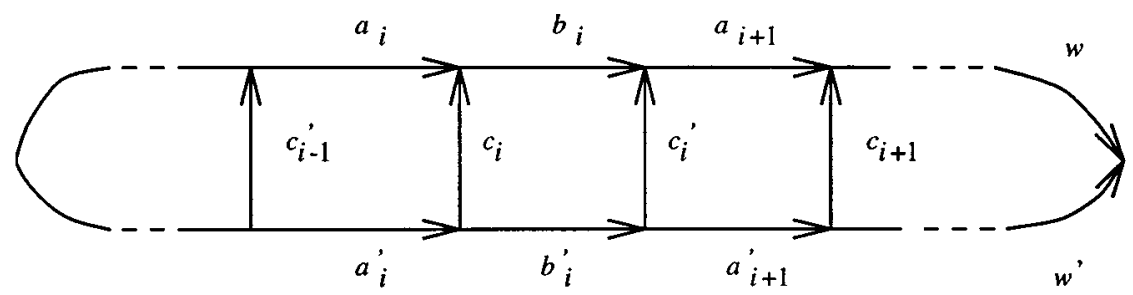

Figure 4. Reduced paths $w$ and $w^{\prime}$ with common end points.

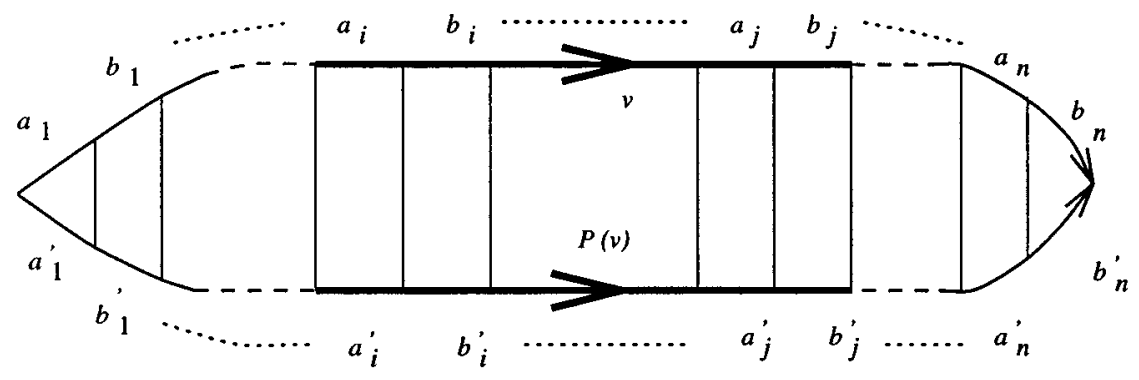

Figure 5. Definition of $P(v)$.

$$
w^{\prime}=a_{1}^{\prime} b_{1}^{\prime} \ldots a_{m}^{\prime} b_{m}^{\prime}
$$

where $a_{1}, b_{n}, a_{1}^{\prime}, b_{m}^{\prime}$ may be empty. Since $\bar{w}=\overline{w^{\prime}}$ and $w$ and $w^{\prime}$ are reduced, we get $n=m$ and there exist $c_{1}, \ldots, c_{n}$ and $c_{0}^{\prime}, c_{1}^{\prime}, \ldots, c_{n}^{\prime}$ in $C$ such that

$$
c_{i-1}^{\prime} a_{i} c_{i}^{-1}=a_{i}^{\prime}, c_{i} b_{i} c_{i}^{-1}=b_{i}^{\prime}
$$

for $1 \leq i \leq n$, where $c_{0}^{\prime}=c_{n}^{\prime}=1$. See Figure 4 .

Let $v$ be a subword of $w$,

$$
v=a_{i} b_{i} \ldots a_{j} b_{j}
$$

where $a_{i}, b_{j}$ may be empty. We define a subword of $w^{\prime}$, denoted by $P(v)$ (see Figure 5), by

$$
P(v)=a_{i}^{\prime} b_{i}^{\prime} \ldots a_{j}^{\prime} b_{j}^{\prime} .
$$

Let $v^{\prime}$ be a subword of $w^{\prime}$. If $v^{\prime}$ is a subword of $P(v)$, we say $v$ covers $v^{\prime}$. If $v^{\prime}=P(v)$, then we say $v$ faces $v^{\prime}$.

Since $|C \backslash A / C| \geq 3$, there exist elements $a_{1}, a_{2} \in A$ such that $a_{1}, a_{2} \in A \backslash C$ and $a_{2} \notin C a_{1} C$. Taking an element $b \in B \backslash C$, we define words $w_{i}, 0 \leq i<\infty$, by

$$
\begin{aligned}
w_{i}= & \left(a_{1} b\right)^{10^{i}}\left(a_{1}^{-1} b^{-1}\right)^{10^{i}}\left(a_{2} b\right)^{10^{i}}\left(a_{2}^{-1} b^{-1}\right)^{10^{i}} \\
& \left(a_{1} b\right)^{4 \cdot 10^{i}}\left(a_{1}^{-1} b^{-1}\right)^{4 \cdot 10^{i}}\left(a_{2} b\right)^{4 \cdot 10^{i}}\left(a_{2}^{-1} b^{-1}\right)^{4 \cdot 10^{i}} .
\end{aligned}
$$

We write the subword $\left(a_{1} b\right)^{4 \cdot 10^{i}}$ by $w_{i}(1,+)$ and the subword $\left(a_{1}^{-1} b^{-1}\right)^{4 \cdot 10^{i}}$ by $w_{i}(1,-)$.

Lemma 4.1. The words $w_{i}, 0 \leq i<\infty$, satisfy the following properties.

(1) For all $i \geq 0$ and all $n \geq 1, w_{i}^{n}$ is reduced.

(2) For all $i \geq 0,\left|w_{i}\right|=40 \cdot 10^{i}$ and $\left|w_{i}(1, \pm)\right|=8 \cdot 10^{i}$.

(3) For all $i \geq 0, \overline{w_{i}} \in[G, G]$. 
Proof. (1) is clear from the way we chose $a_{1}, a_{2}$ and $b$. (2) is obvious. Note that $\overline{\left(a_{i} b\right)^{p}\left(a_{i}^{-1} b^{-1}\right)^{p}} \in[G, G]$ for $i=1,2$ and all $p \geq 1$. This implies (3).

Lemma 4.2. For any pair of paths having a common starting point and a common finishing point, the following properties hold for their subpaths.

(1) $a_{1}$ cannot face $a_{2}$.

(2) For all $i \geq 0, w_{i}^{2}$ cannot cover $w_{i}^{-1}$.

(3) For all $i<j, w_{j}(1,+)$ cannot cover $w_{i}$ and $w_{j}(1,-)^{-1}$ cannot cover $w_{i}$.

(4) For all $k>0$ and all $i<j$, $w_{i}^{k}$ cannot cover $w_{j}$ nor $w_{j}^{-1}$.

Proof. (1) If $a_{1}$ faces $a_{2}$ in some pair of paths, then there exist $c_{1}, c_{2} \in C$ such that $c_{1} a_{1} c_{2}=a_{2}$; thus $a_{2} \in C a_{1} C$, which contradicts our choice of $a_{1}$ and $a_{2}$.

(2) We show this claim for $i=0$;

$$
w_{0}=a_{1} b a_{1}^{-1} b^{-1} a_{2} b a_{2}^{-1} b^{-1}\left(a_{1} b\right)^{4}\left(a_{1}^{-1} b^{-1}\right)^{4}\left(a_{2} b\right)^{4}\left(a_{2}^{-1} b^{-1}\right)^{4} .
$$

We denote the order of the elements labeled by $a_{1}^{ \pm 1}$ or $a_{2}^{ \pm 1}$ in $w_{0}$ by $W_{0}$ in the following way:

$$
W_{0}=1 \overline{1} 2 \overline{2} 1111 \overline{1111} 2222 \overline{2222},
$$

where the symbols $1,2, \overline{1}$ and $\overline{2}$ are for $a_{1}, a_{2}, a_{1}^{-1}$ and $a_{2}^{-1}$, respectively. We put

$$
W_{0}^{-1}=2222 \overline{2222} 1111 \overline{1111} 2 \overline{2} 1 \overline{1}
$$

to represent $w_{0}^{-1}$. To argue by contradiction, assume $w_{0}^{2}$ covers $w_{0}^{-1}$. Then $W_{0}^{2}$ covers $W_{0}^{-1}$. For each conceivable position for $W_{0}^{2}$ covering $W_{0}^{-1}$, one can find at least one of 1's, 2's, $\overline{1}$ 's, or $\overline{2}$ 's in $W_{0}^{-1}$ facing some $2,1, \overline{2}, \overline{1}$, respectively, in $W_{0}^{2}$. See Figure 6 . This implies that there is a pair of subpaths labelled by $a_{1}$ and $a_{2}$ which faces each other in $w_{0}^{2}$ and $w_{0}^{-1}$, which contradicts (1). We showed (2) for $i=0$. A similar argument applies to $w_{i}$ for each $i \geq 1$. (3) Observe that $w_{j}(1,+)$ consists of $a_{1}$ and $b$, while $w_{i}$ contains $a_{2}$. If $w_{j}(1,+)$ covers $w_{i}$, then each $a_{2}$ in $w_{i}$ faces some $a_{1}$ in $w_{j}(1,+)$, which contradicts $(1)$. The same argument applies to $w_{j}(1,-)^{-1}$.

(4) Assume that $w_{i}^{k}$ covers $w_{j}$ for some $i<j$ and some $k>0$. Then $w_{j}(1,+)$ covers some $w_{i}$ of $w_{i}^{k}$ since $2\left|w_{i}\right| \leq\left|w_{j}(1,+)\right|$, which contradicts (3). Assume that $w_{i}^{k}$ covers $w_{j}^{-1}$ for some $i<j$ and some $k>0$. Then $w_{j}(1,-)^{-1}$ covers some $w_{i}$ of $w_{i}^{k}$, which contradicts (3) as well.

Lemma 4.3. The words $w_{i}, 0 \leq i<\infty$, satisfy the following properties.

(1) For all $n \geq 1$ and all $i \geq 0$, we have $c_{w_{i}}\left(w_{i}^{n}\right)=n$.

(2) For all $n \geq 1$ and all $i \geq 0$, we have $c_{w_{i}^{-1}}\left(w_{i}^{n}\right)=0$.

(3) For all $j>i \geq 0$ and all $n \geq 1$, we have $c_{w_{j}}\left(w_{i}^{n}\right)=0$.

(4) For all $j>i \geq 0$ and all $n \geq 1$, we have $c_{w_{j}^{-1}}\left(w_{i}^{n}\right)=0$.

Proof. (1) Since $w_{i}^{n}$ is reduced, $w_{i}^{n}$ is a geodesic. We get $c_{w_{i}}\left(w_{i}^{n}\right) \geq\left|w_{i}^{n}\right|_{w_{i}}=n$. On the other hand, $c_{w_{i}}\left(w_{i}^{n}\right) \leq\left|\overline{w_{i}^{n}}\right| /\left|w_{i}\right|=n$. Thus $c_{w_{i}}\left(w_{i}^{n}\right)=n$.

(2) To show $c_{w_{i}^{-1}}\left(w_{i}^{n}\right)=0$ by contradiction, assume $c_{w_{i}^{-1}}\left(w_{i}^{n}\right)>0$. Let $\alpha$ be a realizing geodesic of $c_{w_{i}^{-1}}$ at $w_{i}^{n}$. Then $|\alpha|_{w_{i}^{-1}}>0$. Fix a subword labeled by $w_{i}^{-1}$ in $\alpha$. Since $w_{i}^{n}$ and $\alpha$ are reduced, there is a subword of $w_{i}^{n}$ labeled by $w_{i}^{2}$ which covers the $w_{i}^{-1}$ in $\alpha$. But $w_{i}^{2}$ cannot cover $w_{i}^{-1}$ by Lemma 4.2(2). We get a contradiction. See Figure 7.

(3) To show the claim by contradiction, assume $c_{w_{j}}\left(w_{i}^{n}\right)>0$ for some $i<j$ and $n$. Take a realizing geodesic $\alpha$ of $c_{w_{j}}$ at $w_{i}^{n}$. Then $|\alpha|_{w_{j}}>0$. Fix some subword 
$W_{0}^{2}: 1 \overline{1} 2 \overline{2} 1111 \overline{1} \overline{1} \overline{1} \overline{1} 2222 \overline{2} \overline{2} \overline{2} \overline{2} 1 \overline{1} 2 \overline{2} 11111 \overline{1} \overline{1} \overline{1} \overline{1} 2222 \overline{2} \overline{2} \overline{2} \overline{2}$ $2222 \overline{2} \overline{2} \overline{2} 1111 \overline{1} \overline{1} \overline{1} \overline{1} 2 \overline{2} 1 \overline{1}$

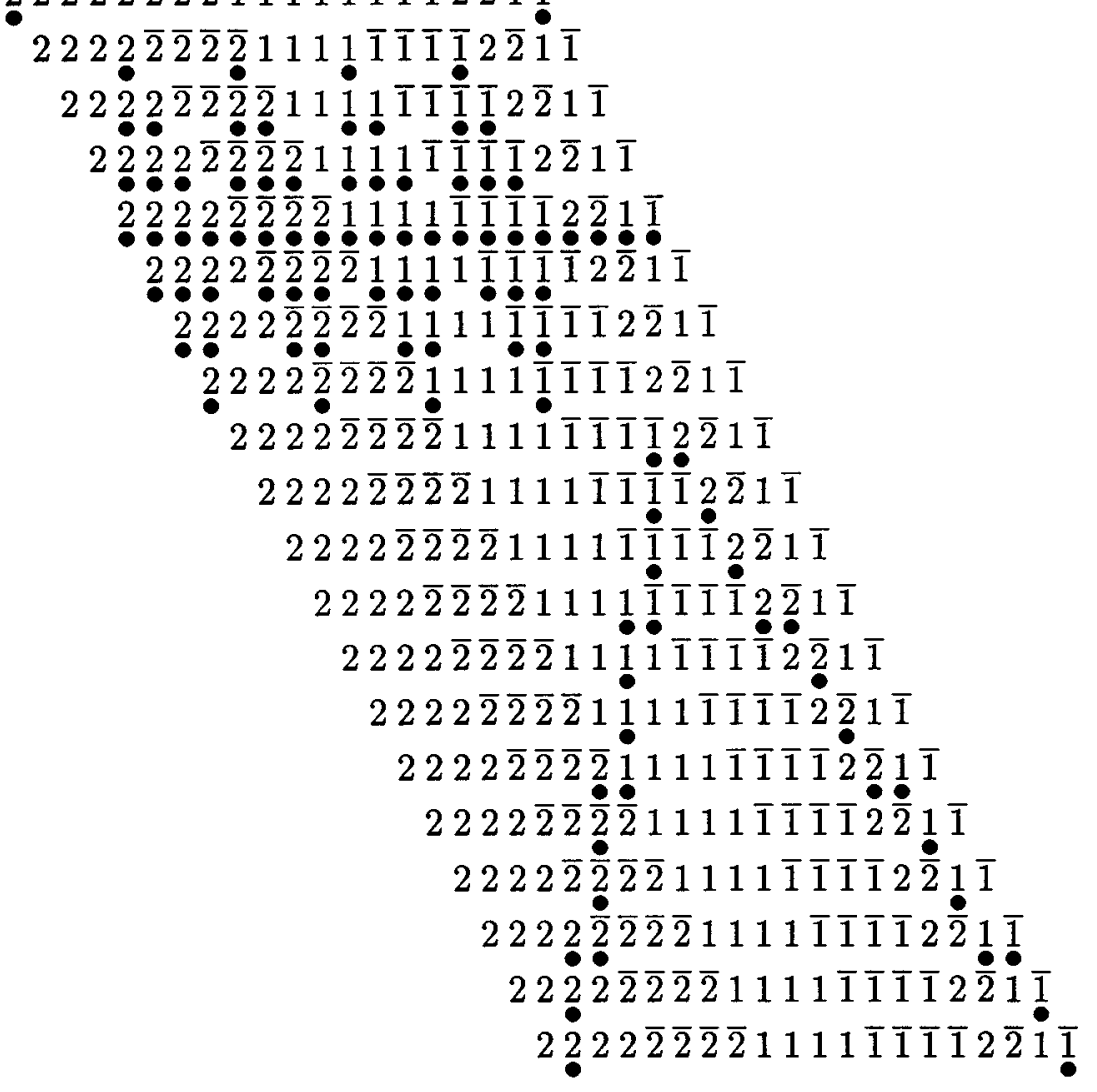

Figure $6 . W_{0}^{2}$ cannot cover $W_{0}^{-1}$. The first row is $W_{0}^{2}$. The second to the last rows describe all the possible positions for $W_{0}^{-1}$. We mark all the "illegal" pairs of $(1,2)$ or $(\overline{1}, \overline{2})$ by $\bullet$. In each position for $W_{0}^{-1}$, we find at least one illegal pair, which is a contradiction.

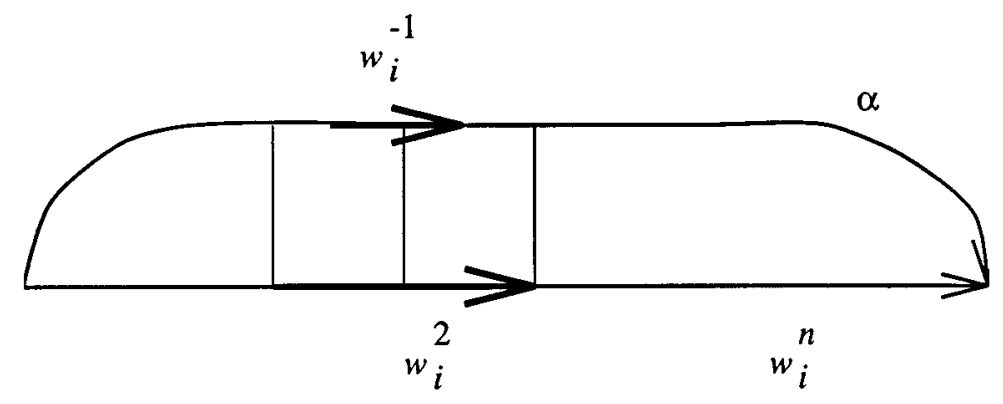

Figure 7. $w_{i}^{2}$ cannot cover $w_{i}^{-1}$. 
labeled by $w_{j}$ in $\alpha$. Since $\alpha$ and $w_{i}^{n}$ are reduced, there exists a subword labeled by $w_{i}^{k}$ in $w_{i}^{n}$ which covers the $w_{j}$ in $\alpha$. But $w_{i}^{k}$ cannot cover $w_{j}$ by Lemma 4.2(4). This is a contradiction.

(4) Similar to (3).

We are in a position to prove Proposition 4.1.

Proof of Proposition 4.1. Let $w_{i}, 0 \leq i<\infty$, be the set of the words in Lemma 4.1 (and 4.2 and 4.3 as well). We show that they are desired words.

(1): Lemma 4.3(1) and (2) imply $h_{w_{i}}\left(w_{i}^{n}\right)=n$ since $h_{w_{i}}=c_{w_{i}}-c_{w_{i}^{-1}}$.

(2): By Lemma 4.3(3) and (4), we obtain $h_{w_{j}}\left(w_{i}^{n}\right)=0$ for $i<j$.

(3): For a homomorphism $\phi: G \rightarrow \mathbb{R}$, we get $\phi\left(\overline{w_{i}}\right)=0$ since $\overline{w_{i}} \in[G, G]$ by Lemma 4.1(3).

(4): By Lemma 4.1(1), $w_{i}^{2}$ is reduced for all $i \geq 0$.

(5): Clear from Lemma 4.1(2).

\section{Proofs of Theorem 1.1 And Corollaries $1.1,1.2$ and 1.3}

Proof of Theorem 1.1. Let $w_{i}, 0 \leq i<\infty$, be the words in Proposition 4.1. Note that $h_{w_{i}}$ is an element of $C^{1}(G ; \mathbb{Z})$. For each $g \in G$, there are only finitely many words $w_{i}$ such that $h_{w_{i}}(g) \neq 0$. This follows from Lemma 2.2 and Proposition 4.1(5). Therefore, if $\left(a_{i}\right)_{i} \in l^{1}$, then $\sum_{i=1}^{\infty} a_{i} h_{w_{i}}$ is also well-defined as an element of $C^{1}(G ; \mathbb{R})$ since this is in fact a finite sum for each $g \in G$. By the same reason, $\sum_{i=1}^{\infty} a_{i} \delta h_{w_{i}}$ is a well-defined cocycle, and the following equality holds.

$$
\delta\left(\sum_{i=1}^{\infty} a_{i} h_{w_{i}}\right)=\sum_{i=1}^{\infty} a_{i} \delta h_{w_{i}} .
$$

Further, since cocycles $\delta h_{w_{i}}, 0 \leq i<\infty$, have the same bound by Proposition 3.1, $\sum_{i} a_{i} \delta h_{w_{i}}$ is bounded. We get a real linear map

$$
\omega: l^{1} \rightarrow H_{b}^{2}(G ; \mathbb{R})
$$

which sends $\left(a_{i}\right)_{i}$ to the cohomology class of $\sum_{i} a_{i} \delta h_{w_{i}}$. In order to show $\omega$ is injective, suppose $\omega\left(\left(a_{i}\right)\right)=0$. Then

$$
\delta\left(\sum_{i=0}^{\infty} a_{i} h_{w_{i}}\right)=\delta b
$$

for some $b \in C_{b}^{1}(G ; \mathbb{R})$. This means

$$
\sum_{i} a_{i} h_{w_{i}}-b=\phi
$$

for some homomorphism $\phi: G \rightarrow \mathbb{R}$. Applying this to $\overline{w_{0}^{n}} \in G$, we find

$$
a_{0} n-b\left(\overline{w_{0}^{n}}\right)=\phi\left(\overline{w_{0}^{n}}\right)=0,
$$

for all $n \geq 1$ by Proposition 4.1. Since $b$ is bounded, $a_{0}=0$. Similarly, $a_{i}=0$ for each $i \geq 1$. Thus $\omega$ is injective. It is well-known that the cardinality of the dimension of $l^{1}$ as a vector space is continuum.

Proof of Corollary 1.1. Without loss of generality, we may assume $|A| \geq 3$. Since $C=\{1\}$, we have $|C \backslash A / C|=|A| \geq 3$. Apply Theorem 1.1.

Proof of Corollary 1.2. Since $|C|<\infty$ and $|A|=\infty$, we have $|C \backslash A / C|=\infty$. Apply Theorem 1.1. 
Proof of Corollary 1.3. Since $A$ is abelian, we have $|C \backslash A / C|=|A / C| \geq 3$. Apply Theorem 1.1.

\section{Quasi homomorphisms ON $A *_{C, \varphi}$}

Let $G=A *_{C, \varphi}=\left\langle A, t ; c=t^{-1} \varphi(c) t\right.$ for all $\left.c \in C\right\rangle$. Suppose $|A / C| \geq 2$ and $|A / \varphi(C)| \geq 2$. Let $g$ be

$$
g=a_{1} t^{n_{1}} a_{2} t^{n_{2}} \ldots a_{I} t^{n_{I}} a_{I+1},
$$

with $a_{i} \in A$ and $n_{i} \neq 0$, where $a_{1}, a_{I+1}$ may be empty. Suppose that for all $i$ $(1 \leq i \leq I-1)$, the following conditions (i) and (ii) are satisfied:

(i) If $n_{i}>0$ and $n_{i+1}<0$, then $a_{i+1} \notin C$.

(ii) If $n_{i}<0$ and $n_{i+1}>0$, then $a_{i+1} \notin \varphi(C)$.

Then we say $g$ is reduced.

The following fact is known as Britton's lemma.

Lemma 6.1 (Britton, $[\mathrm{LS}]$ ). Suppose $1 \leq$ I. If $g$ is reduced, then $g \neq 1$ in $G$.

As an application of Britton's lemma, we have the following lemma.

Lemma 6.2. Let

$$
\begin{gathered}
g=a_{1} t^{n_{1}} a_{2} t^{n_{2}} \ldots a_{I} t^{n_{I}} a_{I+1}, \quad a_{i} \in A, \\
h=b_{1} t^{m_{1}} b_{2} t^{m_{2}} \ldots b_{J} t^{m_{J}} b_{J+1}, \quad b_{j} \in A,
\end{gathered}
$$

be reduced with $n_{i}= \pm 1, m_{j}= \pm 1$. If $g=h$ in $G$, then

$$
I=J, n_{1}=m_{1}, \ldots, n_{I}=m_{I}
$$

and elements

$$
\begin{gathered}
a_{I+1} b_{I+1}^{-1}, t^{n_{I}} a_{I+1} b_{I+1}^{-1} t^{-m_{I}}, a_{I} t^{n_{I}} a_{I+1} b_{I+1}^{-1} t^{-m_{I}} b_{I}^{-1}, \ldots \\
\ldots, t^{n_{1}} a_{2} t^{n_{2}} a_{3} \ldots a_{I+1} b_{I+1}^{-1} \ldots b_{3}^{-1} t^{-m_{2}} b_{2}^{-1} t^{-m_{1}}
\end{gathered}
$$

are in $C$ or $\varphi(C)$, in particular, in $A$.

Proof. Without loss of generality, we may assume $J \leq I$. Since $h$ is reduced, $h^{-1}=b_{J+1}^{-1} t^{-m_{J}} b_{J}^{-1} t^{-m_{J-1}} \ldots b_{1}^{-1}$ is reduced as well. Set

$$
d_{I+1}=a_{I+1} b_{J+1}^{-1} ;
$$

then we have $d_{I+1} \in A$ and

$$
g h^{-1}={ }_{G}\left(a_{1} t^{n_{1}} a_{2} t^{n_{2}} \ldots a_{I} t^{n_{I}}\right) d_{I+1}\left(t^{-m_{J}} b_{J}^{-1} t^{-m_{J-1}} \ldots b_{1}^{-1}\right),
$$

where " ${ }_{G}$ " means that the right- and left-hand sides of the equality represent a common element in $G$. Since $g h^{-1}=_{G} 1$, the word on the right-hand side of the above equality is not reduced by Britton's lemma. But, since $a_{1} t^{n_{1}} a_{2} t^{n_{2}} \ldots a_{I} t^{n_{I}}$ and $t^{-m_{J}} b_{J}^{-1} t^{-m_{J-1}} \ldots b_{1}^{-1}$ are reduced, it follows that

$$
n_{I}=m_{J}=1(\text { or }-1), \quad d_{I+1} \in C(\text { or } \varphi(C), \text { respectively), }
$$

and $t^{n_{I}} d_{I+1} t^{-m_{J}} \in \varphi(C)$ (or $C$, respectively).

Set

$$
c_{I}=t^{n_{I}} d_{I+1} t^{-m_{J}}, d_{I}=a_{I} c_{I} b_{J}^{-1} .
$$

Clearly $d_{I} \in A$ and

$$
g h^{-1}={ }_{G}\left(a_{1} t^{n_{1}} a_{2} t^{n_{2}} \ldots a_{I-1} t^{n_{I-1}}\right) d_{I}\left(t^{-m_{J-1}} b_{J-1}^{-1} t^{-m_{J-2}} \ldots b_{1}^{-1}\right) .
$$




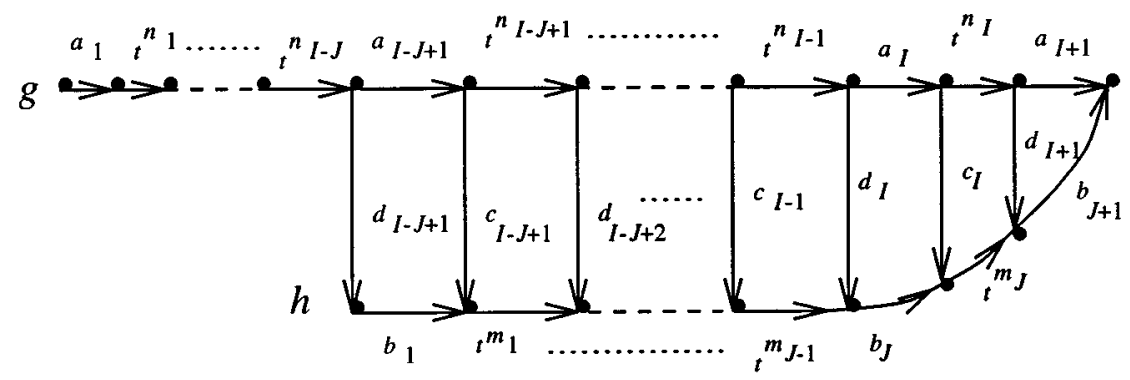

Figure 8. This illustrates Lemma 6.2. Elements $d_{i}, c_{i}$ are in $A$ for all $i$ with $I-J+1 \leq i \leq I$. If $J<I$, then it contradicts Britton's lemma.

By Britton's lemma, the word on the right-hand side is not reduced. Thus

$$
\begin{gathered}
n_{I-1}=m_{J-1}=1(\text { or }-1), \\
d_{I} \in C(\text { or } \varphi(C), \text { respectively }),
\end{gathered}
$$

and

$$
c_{I-1}=t^{n_{I-1}} d_{I} t^{-m_{J-1}} \in \varphi(C) \text { (or } C \text {, respectively). }
$$
by

We define elements $d_{I-1}, d_{I-2}, \ldots, d_{I-J+2}$ and $c_{I-1}, c_{I-2}, \ldots, c_{I-J+1}$ inductively

$$
c_{i}=t^{n_{i}} d_{i+1} t^{-m_{J-I+i}}, \quad d_{i}=a_{i} c_{i} b_{J-I+i}^{-1} .
$$

See Figure 8. Using Britton's lemma repeatedly, we have

$$
n_{I-J+j}=m_{j}, \quad 1 \leq j \leq J,
$$

and

$$
d_{I+1}, d_{I}, \ldots, d_{I-J+2}, c_{I}, c_{I-1}, \ldots, c_{I-J+1} \in C \text { or } \varphi(C) .
$$

Set

$$
d_{I-J+1}=a_{I-J+1} c_{I-J+1} b_{1}^{-1} .
$$

Clearly $d_{I-J+1} \in A$. To complete the proof, it suffices to show $J=I$. In order to show this by contradiction, suppose $J<I$. Then

$$
g h^{-1}={ }_{G} a_{1} t^{n_{1}} a_{2} \ldots t^{n_{I-J-1}} a_{I-J} t^{n_{I-J}} d_{I-J+1} .
$$

The word on the right-hand side is reduced. This contradicts Britton's lemma, since $g h^{-1}={ }_{G} 1$. We get $I=J$.

We take $\{t\} \cup A \backslash\{1\}$ as a set of generators of $G$ and write the Cayley graph of $G$ for this set by $\Gamma$.

Lemma 6.3. If $\alpha$ is a geodesic in $\Gamma$, then it is reduced.

Proof. If a path $\alpha$ is not reduced, then we can make it shorter using a relation $t c t^{-1}=\varphi(c)$; hence $\alpha$ is not a geodesic.

Remark. Compare Lemma 6.3 with Lemma 3.1. A reduced path is not always a geodesic in this case. For example, the left-hand side of $\varphi(c)^{-1} t c=t$ for $c \in C \backslash\{1\}$ is reduced but not a geodesic.

Lemma 6.4. Let $\alpha$ be a path and $w$ a word. If $w^{2}$ is reduced, then there is a reduced path $\beta$ which realizes $c_{w}$ at $\alpha$. 
Proof. Take a path $\beta$ which realizes $c_{w}$ at $\alpha$ such that $|\beta|_{w}$ is minimal among all the realizing paths. Then an argument similar to the proof of Lemma 3.2 shows $\beta$ is reduced.

Lemma 6.5. Suppose $w^{2}$ is reduced. Let $\alpha, \beta$ be paths starting at 1 . Then we have

$$
\begin{gathered}
\left|c_{w}(\alpha)-c_{w}(\beta)\right| \leq 2\left|\overline{\alpha^{-1} \beta}\right| \\
\left|h_{w}(\alpha)-h_{w}(\beta)\right| \leq 4\left|\overline{\alpha^{-1} \beta}\right| .
\end{gathered}
$$

Proof. Similar to Lemmas 3.3 and 3.4.

Lemma 6.6. Suppose $w^{2}$ is reduced. Let $\alpha$ be a reduced path. If $\alpha=\alpha_{1} \alpha_{2}$, then

$$
\left|h_{w}(\alpha)-h_{w}\left(\alpha_{1}\right)-h_{w}\left(\alpha_{2}\right)\right| \leq 10 .
$$

Proof. Similar to Lemma 3.7.

Proposition 6.1. Suppose $w^{2}$ is reduced. Then we have

$$
\left|\delta h_{w}\right| \leq 78 \text {. }
$$

Proof. The outline is similar to the proof of Proposition 3.1. Let $x, y$ be elements in $G$. It suffices to show $\left|h_{w}(x y)-h_{w}(x)-h_{w}(y)\right| \leq 78$. Take reduced paths $\alpha, \beta, \gamma$ with $\bar{\alpha}=x, \bar{\beta}=y, \bar{\gamma}=x y$. Since $\overline{\alpha \beta}=\bar{\gamma}$ and $\alpha, \beta, \gamma$ are reduced, by Lemmas 6.1 and 6.2 , there exist subdivisions of $\alpha, \beta, \gamma$ such that

$$
\alpha=\alpha_{1} \alpha_{2} \alpha_{3}, \beta=\beta_{1} \beta_{2} \beta_{3}, \gamma=\gamma_{1} \gamma_{2} \gamma_{3},
$$

and that $\overline{\gamma_{1}^{-1} \alpha_{1}}=c_{1}, \overline{\alpha_{3} \beta_{1}}=c_{2}, \overline{\beta_{3} \gamma_{3}^{-1}}=c_{3}$ for some $c_{1}, c_{2}, c_{3} \in C \cup \varphi(C)$, and $\overline{\alpha_{2}}, \overline{\beta_{2}}, \overline{\gamma_{2}} \in A$. By Lemma 6.6,

$$
\left|h_{w}(\alpha)-h_{w}\left(\alpha_{1}\right)-h_{w}\left(\alpha_{2}\right)-h_{w}\left(\alpha_{3}\right)\right| \leq 20 .
$$

Since $\left|h_{w}\left(\alpha_{2}\right)\right| \leq 2,\left|h_{w}(\alpha)-h_{w}\left(\alpha_{1}\right)-h_{w}\left(\alpha_{3}\right)\right| \leq 22$. Similarly,

$$
\left|h_{w}(\beta)-h_{w}\left(\beta_{1}\right)-h_{w}\left(\beta_{3}\right)\right| \leq 22,\left|h_{w}(\gamma)-h_{w}\left(\gamma_{1}\right)-h_{w}\left(\gamma_{3}\right)\right| \leq 22 .
$$

By an argument similar to the proof of Proposition 3.1,

$$
\left|h_{w}(x)+h_{w}(y)-h_{w}(x y)\right|=\left|h_{w}(\alpha)+h_{w}(\beta)-h_{w}(\gamma)\right| \leq 78 .
$$

7. Choice of Words For $A *_{C, \varphi}$

Let $G=A *_{C, \varphi}$ with $|A / C| \geq 2,|A / \varphi(C)| \geq 2$.

Lemma 7.1. Let $w$ be

$$
w=t^{n_{1}} a_{1} t^{n_{2}} a_{2} \ldots t^{n_{I}} a_{I}
$$

such that $a_{i} \in A \backslash\{1\}$ and $n_{i} \in \mathbb{Z} \backslash\{0\}$ for all $i$ with $1 \leq i \leq I$. We denote the set of the following conditions (1.1),.., (1.4) by Condition I and the set (2.1),..., (2.4) by Condition II.

(1.1) $0<n_{1}, n_{3}, n_{5}, \ldots$

(1.2) $0>n_{2}, n_{4}, n_{6}, \ldots$.

(1.3) $a_{1}, a_{3}, a_{5}, \cdots \notin C$.

(1.4) $a_{2}, a_{4}, a_{6}, \cdots \notin \varphi(C)$.

(2.1) $0>n_{1}, n_{3}, n_{5}, \ldots$.

(2.2) $0<n_{2}, n_{4}, n_{6}, \ldots$ 
(2.3) $a_{1}, a_{3}, a_{5}, \cdots \notin \varphi(C)$.

(2.4) $a_{2}, a_{4}, a_{6}, \cdots \notin C$.

If either the Condition I or II holds, then $w$ is a geodesic in $\Gamma$.

Proof. Suppose Condition I holds. The other case is similar. Clearly $w$ is reduced. Let $\gamma$ be a geodesic with $\bar{w}=\bar{\gamma}$. Then by Lemma $6.3, \gamma$ is reduced. By Lemma 6.2 , we have

$$
\gamma=b_{0} \tau_{1} b_{1} \tau_{2} b_{2} \ldots \tau_{I} b_{I}
$$

such that $b_{i} \in A \backslash\{1\}$ for $1 \leq i \leq I-1, b_{0}, b_{I}$ are in $A \backslash\{1\}$ or empty, and

$$
\begin{aligned}
\tau_{i} & =t b_{i, 1} t b_{i, 2} \ldots t b_{i, n_{i}-2} t b_{i, n_{i}-1} t, \text { if } i \text { is odd, } \\
\tau_{i} & =t^{-1} b_{i, 1} t^{-1} b_{i, 2} \ldots t^{-1} b_{i, n_{i}-2} t^{-1} b_{i, n_{i}-1} t^{-1}, \text { if } i \text { is even, }
\end{aligned}
$$

where $b_{i, j}$ are in $A \backslash\{1\}$ or empty for $1 \leq i \leq I$ and $1 \leq j \leq n_{i}-1$.

We claim that $b_{I}$ is not empty. To show this by contradiction, assume $b_{I}$ is empty. Then

$$
\begin{aligned}
& w \gamma^{-1}=\ldots t a_{I} t^{-1} \ldots, \text { if } I \text { is odd } \\
& w \gamma^{-1}=\ldots t^{-1} a_{I} t \ldots, \text { if } I \text { is even. }
\end{aligned}
$$

Since $w$ and $\gamma^{-1}$ are reduced, $w \gamma^{-1}$ is reduced by (1.3) if $I$ is odd or by (1.4) if $I$ is even. Then it follows from Britton's lemma that $\overline{w \gamma^{-1}} \neq 1$. This is a contradiction. We get $b_{I}$ is not empty. Thus

$$
|\gamma| \geq \sum_{i=1}^{I}\left|\tau_{i}\right|+I \geq \sum_{i=1}^{I}\left|n_{i}\right|+I=|w| .
$$

We have the first inequality because $b_{1}, \ldots, b_{I}$ are not empty, and the second one since the number of $t$ 's in $\tau_{i}$ is $n_{i}$ for $1 \leq i \leq I$. Since $\gamma$ is a geodesic, we have $|\gamma|=|w|$; hence $w$ is a geodesic.

Lemma 7.2. Take some elements $g \in A \backslash C$ and $h \in A \backslash \varphi(C)$ and fix them. Let $w_{i}, 0 \leq i<\infty$, be words such that

$$
w_{i}=t^{10^{i}} g t^{-10^{i}} h t^{10^{i}} g^{-1} t^{-10^{i}} h^{-1} t^{2 \cdot 10^{i}} g t^{-2 \cdot 10^{i}} h t^{3 \cdot 10^{i}} g^{-1} t^{-3 \cdot 10^{i}} h^{-1} .
$$

Then the words $w_{i}$ satisfy the following properties.

(1) For all $i \geq 0$ and all $n \geq 1$, we have $c_{w_{i}}\left(w_{i}^{n}\right)=n$.

(2) For all $i \geq 0$ and all $n \geq 1$, we have $c_{w_{i}^{-1}}\left(w_{i}^{n}\right)=0$.

(3) For all $j>i \geq 0$ and all $n \geq 1$, we have $c_{w_{j}^{ \pm 1}}\left(w_{i}^{n}\right)=0$.

(4) For all $i \geq 0$, we have $\overline{w_{i}} \in[G, G]$.

(5) For all $i \geq 0$, we have $\left|w_{i}\right|=8+14 \cdot 10^{i}$.

Proof. (1) By Lemma 7.1, $w_{i}^{n}$ is a geodesic. Thus $c_{w_{i}}\left(w_{i}^{n}\right)=n$.

(2) In order to show $c_{w_{0}^{-1}}\left(w_{0}^{n}\right)=0$ for all $n \geq 1$ by contradiction, suppose $c_{w_{0}^{-1}}\left(w_{0}^{n}\right)>0$ for some $n$. By Lemma 6.4, take a reduced path $\alpha$ such that $\bar{\alpha}=\overline{w_{0}^{n}}$ and $|\alpha|_{w_{0}^{-1}}>0$. We describe the order of $t^{\text {'s }}$ and $t^{-1}$ 's appearing in $w_{0}$ by $W_{0}$,

$$
W_{0}=+-+-++--+++--- \text {, }
$$

where + stands for $t,-$ for $t^{-1}$, and we disregard $g^{ \pm 1}, h^{ \pm 1}$ in $w_{0}$. Then $W_{0}^{n}$ represents $w_{0}^{n}$. In the same fashion let $\Lambda$ denote the order of $t^{\prime}$ s and $t^{-1}$ 's in $\alpha$. 
Applying Lemma 6.2 to $\alpha$ and $w_{0}^{n}$, we get $W_{0}^{n}=\Lambda . \Lambda$ contains $W_{0}^{-1}$ as a subpiece since $|\alpha|_{w_{0}^{-1}}>0$, where

$$
W_{0}^{-1}=+++---++--+-+- \text {. }
$$

But it is easy to check that $W_{0}^{n}$ cannot contain $W_{0}^{-1}$ as a subset. We get a contradiction. Hence $c_{w_{0}^{-1}}\left(w_{0}^{n}\right)=0$. Similarly, we get $c_{w_{i}^{-1}}\left(w_{i}^{n}\right)=0$ for each $i \geq 1$ and all $n \geq 1$.

(3) Let $W_{i}, 1 \leq i<\infty$, be the order of $t^{\prime}$ s and $t^{-1}$ 's in $w_{i}$. To show $c_{w_{j}}\left(w_{i}^{n}\right)=0$ for all $n \geq 1$ and all $j>i>0$ by contradiction, assume $c_{w_{j}}\left(w_{i}^{n}\right)>0$ for some $n \geq 1$ and some $j>i>0$. Then, by our assumption, $W_{j}$ must be contained in $W_{i}^{n}$ as a subword. This is a contradiction since $W_{j}$ has $3 \cdot 10^{j}$ consecutive $t$ 's, but $W_{i}$ has at most $3 \cdot 10^{i}$ consecutive $t$ 's. Thus we get $c_{w_{j}}\left(w_{i}^{n}\right)=0$. Similarly, $c_{w_{j}^{-1}}\left(w_{i}^{n}\right)=0$.

(4) Set $T=t^{10^{i}}$. Then

$$
w_{i}=[T, g]\left[g h,\left[T, g^{-1}\right]\right]\left[T, g^{-1}\right][g, h]\left[T^{2}, g\right]\left[g h,\left[T^{3}, g^{-1}\right]\right]\left[T^{3}, g^{-1}\right][g, h] ;
$$

thus $\overline{w_{i}} \in[G, G]$.

(5) This is clear.

Proposition 7.1. Let $G=A *_{C, \varphi}$ with $|A / C| \geq 2,|A / \varphi(C)| \geq 2$. There exist words $w_{i}, 0 \leq i<\infty$, which satisfy the following properties.

(1) For all $i \geq 0$ and all $n \geq 1$, we have $h_{w_{i}}\left(w_{i}^{n}\right)=n$.

(2) For all $j>i \geq 0$ and all $n \geq 1$, we have $h_{w_{j}}\left(w_{i}^{n}\right)=0$.

(3) For all $i \geq 0$, we have $\overline{w_{i}} \in[G, G]$.

(4) For all $i \geq 0, w_{i}^{2}$ is reduced.

(5) $\lim _{i \rightarrow \infty}\left|w_{i}\right|=\infty$.

Proof. The words $w_{i}, 0 \leq i<\infty$, in Lemma 7.2 clearly suffice.

\section{Proofs of Theorem 1.2 And Theorem 1.3}

Proof of Theorem 1.2. Theorem 1.2 follows from Proposition 6.1 and 7.1 as Theorem 1.1 did from Proposition 3.1 and 4.1.

Proof of Theorem 1.3. By Stallings' structure theorem [S], we know that $G$ is either (1) $A *_{C} B$ with $|C|<\infty,|A / C| \geq 3$ and $|B / C| \geq 2$,

or

(2) $A *_{C, \varphi}$ with $|C|<\infty$ and $|A / C| \geq 2$.

Suppose the condition(1) holds. If $|A|=\infty$ or $|B|=\infty$, then we have the conclusion by Corollary 1.2. If $|A|<\infty$ and $|B|<\infty$, then $G$ is word-hyperbolic. Since $G$ has infinitely many ends, it is non-elementary. It is known that the conclusion of Theorem 1.3 holds for a non-elementary word-hyperbolic group [EF]. Suppose the condition(2) holds. Then since $|A / C| \geq 2$ and $|C|<\infty$, we have $|A / \varphi(C)| \geq 2$ as well. Apply Theorem 1.2.

\section{REFERENCES}

[BaGh1] J. Barge, E. Ghys, Surfaces et cohomologie bornée, Invent. Math., 92, 1988, 509-526. MR 89e:55015

[BaGh2] J. Barge, E. Ghys, Cocycles bornés et actions de groupes sur les arbres réels, in "Group Theory from a Geometric Viewpoint", World Sci. Pub., New Jersey, 1991, 617-622. MR 93f: 20033

[BaGh3] J. Barge, E. Ghys, Cocycles d'Euler et de Maslov, Math. Ann., 294, no 2, 1992, 235-265. MR 95b:55021 
[Bav] C. Bavard, Longueur stable des commutateurs, L'Enseignement Math., 37, 1991, 109150. MR 92g:20051

[B] R. Brooks, Some remarks on bounded cohomology, Ann. Math. Studies, 97, 1981, 53-63. MR 83a:57038

[BS] R. Brooks, C. Series, Bounded cohomology for surface groups, Topology, 23, no 1, 1984, 29-36. MR 85c:57009

[Br] K.S. Brown, "Cohomology of groups", Springer, New York, 1982. MR 83k:20002

[EF] D.B.A. Epstein, K. Fujiwara, The second bounded cohomology of word-hyperbolic groups, Topology 36, 1997, 1275-1289. MR 98k:20088

[F] K. Fujiwara, The second bounded cohomology of a group acting on a Gromov-hyperbolic space, Proc. London Math Soc. (3) 76, 1998, 70-94. MR 99c:20072

[FO] K. Fujiwara, K. Ohshika, The second bounded cohomology of 3-manifolds, preprint, Univ Tokyo, 1997.

[Gh] E. Ghys, Groupes d'homéomorphismes du cercle et cohomologie bornée, Contemp. Math. 58, AMS, 1987, 81-106. MR 88m:58024

[Gr] R.I. Grigorchuk, Some remarks on bounded cohomology, LMS Lecture Note 204, Cambridge Univ Press, Cambridge, 1995, 111-163. MR 96j:20073

[G] M. Gromov, Volume and bounded cohomology, Publ. Math. IHES, 56, 1982, 5-99. MR 84h: 53053

[I] N.V. Ivanov, Foundations of the theory of bounded cohomology, Zap. Nauchn. Sem. Leningr. Otd. Mat. Inst., 143, 1985, 69-109. MR 87b:53070

[LS] R.C. Lyndon, P.E. Schupp, "Combinatorial Group Theory", Berlin, Springer, 1977. MR $\mathbf{5 8 : 2 8 1 8 2}$

[MaMo] S. Matsumoto, S. Morita, Bounded cohomology of certain groups of homomorphisms, Proc. A.M.S., 94, 1985, 539-544. MR 87e:55006

[Mi] Y. Mitsumatsu, Bounded cohomology and $l^{1}$-homology of surfaces, Topology, 23, 1984, 465-471. MR 86f:57010

[So1] T. Soma, Bounded cohomology of closed surfaces, Topology 36, 1997, 1221-1246. MR 99a:57011

[So2] T. Soma, Bounded cohomology and topologically tame Kleinian groups, Duke Math Jour. 88, 1997, no 2, 357-370. MR 98f:57023

[S] J. Stallings, "Group theory and three-dimensional manifolds", Yale Univ. Press, New Haven, 1971. MR 54:3705

[Y] T. Yoshida, On 3-dimensional bounded cohomology of surfaces, in "Homotopy Theory and Related Topics", Advanced Studies in Pure Math 9, Kinokuniya, Tokyo, 1986, 173176. MR 88m:57032

Department of Mathematics, Keio University, Yokohama, 223 Japan

Current address: Math Institute, Tohoku Univeristy, Sendai, 980-8578, Japan

E-mail address: fujiwara@math.tohoku.ac.jp 